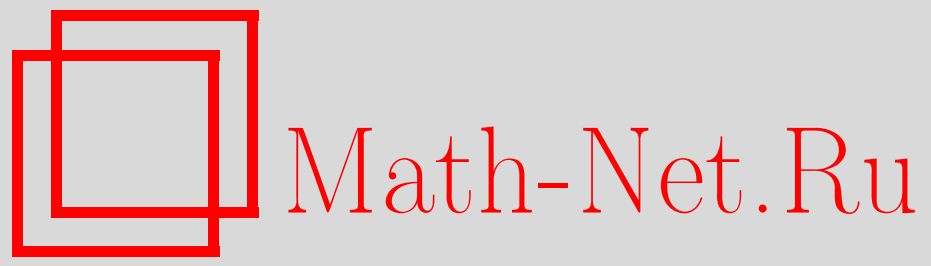

И. М. Дектярев, Равнораспределенность значений при полиномиальных отображениях, Функи. анализ и его прил., 1999, том 33, выпуск 4, 70-73

DOI: https://doi.org/10.4213/faa383

Использование Общероссийского математического портала MathNet.Ru подразумевает, что вы прочитали и согласны с пользовательским соглашением

http://www . mathnet.ru/rus/agreement

Параметры загрузки:

IP : 54.172 .240 .79

26 апреля 2023 г., 15:13:53

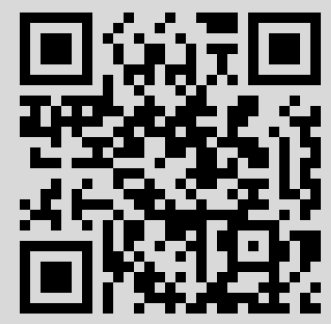


УДК 519.9

\title{
Равнораспределенность значений при полиномиальных отображениях ${ }^{*}$
}

\author{
(C) 1999. И. М. ДЕКТярев
}

Мы будем изучать полиномиальные отображения $F: \mathbb{C}^{n} \rightarrow \mathbb{C}^{n}$, для которых однородные составляющие старших степеней не имеют нетривиальных общих корней. Такие отображения будем называть полиномиальными отображениями обшего вида.

Если $F$ - отображение общего вида, то из $|z| \rightarrow \infty$ следует, что $|F(z)| \rightarrow \infty$. Значит, прообраз любой точки $a \in \mathbb{C}^{n}$, будучи аналитическим множеством в компактной части пространства $\mathbb{C}^{n}$, является конечным, и по теореме Безу количество $\operatorname{deg}(F)$ элементов в нем с учетом кратности равно произведению степеней полиномов, образующих отображение $F$. Будем называть это число степенью отображения $F$.

Пусть для $R>0$ и $\rho>0$ из $|z|>R$ следует $|F(z)|>2 \rho$, и пусть $f$ - голоморфная функция в окрестности замыкания шара радиуса $R$, а $M$ - максимум модуля функции $f$ на этом шаре. В работе [1, с. 69] доказано существование такой константы $L$, зависящей только от $M, \operatorname{deg}(F)$ и $\rho$, что для любых $w_{1}$ и $w_{2}$, принадлежащих шару радиуса $\rho$, хаусдорфово расстояние между множествами $f\left(F^{-1}\left(w_{1}\right)\right)$ и $f\left(F^{-1}\left(w_{2}\right)\right)$ не превосходит величины $L\left|w_{1}-w_{2}\right|^{1 / \operatorname{deg}(F)}$. Отсюда, в частности, следует, что отображение $F$ открыто.

Пространство-образ будем считать естественно вложенным в проективное пространство, которое, в свою очередь, будем рассматривать как проективизацию унитарного $(n+1)$-мерного пространства. На пространстве $\mathbb{C P}^{n}$ зададим $(n, n)$-форму $\omega$ - нормированную унитарно инвариантную форму объема - и через $\mu$ обозначим $(n-1)$-ю внешнюю степень фундаментальной формы стандартной кэлеровой метрики Фубини-Штуди, поделенной на $\pi$. Обозначая через $\alpha$ модуль угла между прямыми, задающими точки $a$ и $w$ проективного пространства, определим, кроме того, функцию

$$
\gamma_{a}(w)=\frac{1}{4 \pi}\left(\ln \frac{1}{\sin ^{2} \alpha}+\sum_{1}^{n-1} \frac{1}{k} C_{n-1}^{k} \operatorname{tg}^{-2 k} \alpha\right)
$$

Непосредственно проверяется, что $d d^{c}\left(\gamma_{a} \mu\right)=\omega$ вне точки $a$. Кроме того (см. $[1$, с. 69$])$, из открытости отображения $F$ следует, что форма $F^{*}\left(\gamma_{a} \mu\right)$ имеет локально абсолютно суммируемые коэффициенты. Это же верно и относительно коэффициентов форм $d F^{*}\left(\gamma_{a} \mu\right)$ и $d^{c} F^{*}\left(\gamma_{a} \mu\right)$.

Лемма 1. Пусть $a=F\left(z_{0}\right), U$ - окрестность точки $z_{0}, \theta$ - произвольная гладкая 1-форма, а $f$ - непрерьвная функция в окрестности $U$. Для любого $\varepsilon>0$ найдется такая окрестность $V \subset U$, что для любой окрестности $W \subset V$, имеющей кусочно гладкую гранииу, выполнено соотношение $\left|\int_{\partial W} \theta \wedge F^{*}\left(\gamma_{a} \mu\right)\right|<\varepsilon$. Если же $z_{0}$ - регулярная точка отображения $F$, то

* Работа выполнена при поддержке Российского фонда фундаментальных исследований, грант 97-01-00050. 
для некоторой окрестности $W \subset U$ с кусочно гладкой границей выполнено соотношение $\left|\int_{\partial W} f d^{c} F^{*}\left(\gamma_{a} \mu\right)+f\left(z_{0}\right)\right|<\varepsilon$.

Пусть $\Phi$ - пространство гладких финитных функций на пространстве $\mathbb{C}^{n}$, и пусть $\psi$ - это форма $(4 \pi)^{-1} \sum_{j=1}^{n} d d^{c} \ln \left(1+\left|z_{j}\right|^{2}\right)$. Определим норму функции $\varphi \in \Phi$ как точную нижнюю грань таких $\lambda>0$, что формы $\lambda \psi+d d^{c} \varphi$ и $\lambda \psi-d d^{c} \varphi$ положительны. Определим функционал $N(F, a)$ как сумму значений функции $\varphi$ на прообразах точки $a$, подсчитанную с учетом кратности. Функционал $T(F)$ определим как интеграл по $\mathbb{C}^{n}$ от формы $\varphi F^{*} \omega$, а функционал $m(F, a)$ - как интеграл по $\mathbb{C}^{n}$ от формы $d d^{c} \varphi \wedge F^{*}\left(\gamma_{a} \mu\right)$. Эти объекты являются в определенном смысле аналогами соответствующих величин теории Неванлинны. Во всяком случае имеет место следующий аналог первой основной теоремы.

ТЕОремА 1. Для любой Функиии $\varphi \in \Phi$ июбого значения а выполнено соотношение $T(F) \varphi=N(F, a) \varphi+m(F, a) \varphi$, причем функциональ, стоящие в правой части, слабо непрерьвно зависят от а.

ДокАЗАтЕльство. Непрерывность функционалов $N(F, a)$ и $m(F, a)$ легко следует из [1, леммы 4 и 5]. Поэтому достаточно доказать теорему для регулярных значений, что можно сделать непосредственными вычислениями, используя теорему Стокса и лемму 1.

СЛЕДСТВИЕ 1. Норма функиионала $T(F)-N(F, a)$ ни при каком значении $а$ не превосходит величиньл $\int_{\mathbb{C}^{n}} \psi \wedge F^{*}\left(\gamma_{a} \mu\right)$.

Используя функцию $\gamma_{a}$ в качестве ядра, можно построить на пространстве $\mathbb{C P}^{n}$ теорию потенциала. При этом получающиеся свойства во многом аналогичны свойствам ньютоновского потенциала. В частности [2, с. 253], если емкость какого-либо множества $D$ равна $\operatorname{Cap}(D)$, то для любого $\delta>0$ найдется такое покрытие этого множества шарами радиусов $r_{1}, r_{2}, \ldots$, что $\sum_{k} r_{k}^{2 n-2+\delta}<$ $L \operatorname{Cap}(D)^{1+\delta /(2 n-2)}$, где константа $L$ зависит только от размерности.

ТЕОрема 2. Пусть компактное множество $D \subset \mathbb{C P}^{n}$ состоит из таких точек а, что норма функиионала $T(F)-N(F, a)$ для этих а больше, чем $A$. Тогда емкость $\mathrm{Cap}(D)$ этого множества не превосходит величинь $(n / A) d_{F}$, где через $d_{F}$ обозначено произведение степеней всех полиномов, образуюших отображение $F$, за исключением полинома, имеюшего наименьшую степень.

ДокАЗАтЕльство. Проинтегрируем оценку из следствия 1 по равновесной мере на множестве $D$ и поменяем местами порядок интегрирования. Мы получим, что $A<(1 / \operatorname{Cap}(D)) \int_{\mathbb{C}^{n}} \psi \wedge F^{*} \mu$, и нам достаточно при любом $j$ установить нужную оценку для интеграла

$$
\frac{1}{4 \pi} \int_{\mathbb{C}^{n}} d d^{c} \ln \left(1+\left|z_{j}\right|^{2}\right) \wedge F^{*} \mu=\frac{1}{4 \pi} \int_{\mathbb{C}}\left(\int_{z_{j}=t} F^{*} \mu\right) d d^{c} \ln \left(1+|t|^{2}\right) .
$$

Внутренний интеграл здесь берется по гиперплоскости $z_{j}=t$, и, согласно теореме Виртингера, он равен умноженному на величину $(n-1) ! / \pi^{n-1}$ объему образа этой гиперплоскости, измеренному в метрике, индуцированной стандартной метрикой Фубини-Штуди. Этот объем, в свою очередь, по теореме Крофтона равен умноженному на величину $\pi^{n-1} /(n-1)$ ! среднему числу пересечений образа этой гиперплоскости с прямыми проективного пространства (усреднение берется по мере, инвариантной на множестве прямых). Покажем, что для почти всех прямых число точек пересечения не превосходит величины $d_{F}$. 
Пусть для определенности среди полиномов $f^{1}, \ldots, f^{n}$, задающих отображение $F$, наименьшую степень имеет первый полином. Рассмотрим в пространстве $\mathbb{C}^{n} \subset \mathbb{C P}^{n}$ векторы $\boldsymbol{a}=\left(0, a^{2}, \ldots, a^{n}\right)$ и $\boldsymbol{b}=\left(1, b^{2}, \ldots, b^{n}\right)$, определяющие прямую $\boldsymbol{a}+s \boldsymbol{b}$. В таком виде можно представить почти любую прямую, и по теореме Бертини почти любая прямая пересекает образ гиперплоскости трансверсально. Количество точек пересечения равно количеству решений системы уравнений $\left\{f^{1}=s, f^{2}=a^{2}+s b^{2}, \ldots, f^{n}=a^{n}+s b^{n}\right\}$. Исключая при помощи первого уравнения параметр $s$ и учитывая, что полином $f^{1}$ имеет наименьшую степень, мы получим необходимый результат, воспользовавшись следуюшим дополнением к теореме Безу: если отображение $F: \mathbb{C}^{n} \rightarrow \mathbb{C}^{n}$ (не обязательно общего вида) задается системой полиномов, то количество изолированных точек (с учетом кратности) в прообразе любой точки $a$ не превосходит величины $\operatorname{deg}(F)$.

СлЕДСТВИЕ 2. Пусть $F_{1}, \ldots, F_{k}, \ldots$ - последовательность полиномиальньцх отображений общего вида, и пусть последовательность $d_{1}, \ldots, d_{k}, \ldots$ наименьших степеней полиномов, входящих в эти отображения, монотонно стремится $к$ бесконечности. Тогда хаусдорфова размерность множества исключительных значений, т. е. тех значений а, для которых норма функиионала $\left(T\left(F_{k}\right)-N\left(F_{k}, a\right)\right) / \operatorname{deg}\left(F_{k}\right)$ не стремится $к$ нулю, не превосходит $2 n-2$.

ДокАЗАТЕльСтво. Зададимся произвольными положительными числами $\varepsilon$ и $\delta$ и для каждого $k=1,2, \ldots$ построим множество $E_{k}$, которое состоит из тех значений $a$, для которых норма функционала $\left(T\left(F_{k}\right)-N\left(F_{k}, a\right)\right) / \operatorname{deg}\left(F_{k}\right)$ больше, чем $k^{-\delta /(4 n-4)}$. Согласно теореме 2 , емкость множества $E_{k}$ не превосходит величины $L_{1} k^{\delta /(4 n-4)} / d_{k}$, где константа $L_{1}$ зависит только от размерности. Каждое такое $E_{k}$ можно покрыть системой шаров, для радиусов которых выполнено соотношение $\sum r_{s}^{2 n-2+\delta}<L_{2} k^{\delta(1+\delta /(2 n-2)) /(4 n-4)} / d_{k}^{1+\delta /(2 n-2)}$. Если выбрать $\delta$ меньшим, чем $(2 n-2) / 3$, и учесть монотонное возрастание целочисленной последовательности $d_{1}, \ldots, d_{k}, \ldots$, то окажется, что выражение в правой части не превосходит $L_{2} k^{-(1+\delta /(6 n-6))}$. Выберем число $k_{0}$ настолько большим, что $L_{2} \sum_{k>k_{0}} k^{-(1+\delta /(6 n-6))}<\varepsilon$. Множество исключительных значений содержится в $\bigcup_{k>k_{0}} E_{k}$ и потому в силу изложенного может быть покрыто системой шаров, радиусы которых удовлетворяют соотношению $\sum r_{s}^{2 n-2+\delta}<\varepsilon$, т. е. $(2 n-2+\delta)$-мерная мера Хаусдорфа этого множества равна нулю.

ЗАмечАние. Результаты, похожие на изложенные выше, были получены в работах $[3,4]$. В частности, в [3] доказано следующее (мы используем термины и обозначения настоящей заметки): пусть $F_{1}, \ldots, F_{k}, \ldots$ - последовательность невырожденных полиномиальных отображений (не обязательно общего вида), и пусть последовательность $d_{1}, \ldots, d_{k}, \ldots$ наибольших (а не наименьших, как у нас) степеней полиномов, входящих в эти отображения, монотонно стремится к бесконечности. Тогда хаусдорфова размерность множества тех значений $a$, для которых норма функционала $\left(T\left(F_{k}\right)-N\left(F_{k}, a\right)\right) / d_{k}^{n}$ не стремится к нулю, не превосходит $2 n-2$. Отметим, однако, что знаменатель в этой формулировке слишком велик, и во многих интересных случаях стремление к нулю обеспечивается именно величиной знаменателя, а не близостью между собой величин, входящих в числитель. Впрочем, это отмечалось и авторами цитируемой работы. В работе [4] изучаются рациональные отображения проективных 
пространств. Однако полученные там условия равнораспределенности являются более жесткими. Для последовательностей отображений, рассмотренных в настоящей заметке, они сводятся к требованию сходимости ряда $\sum d_{k}^{-1}$ (в обозначениях настоящей заметки).

Приводимые ниже примеры показывают, что от налагаемых нами условий на последовательность отображений отказаться нельзя.

ПримеР 1. Рассмотрим последовательность $\left\{w_{1}=z_{1}^{k}, w_{2}=z_{2}\right\}$ отображений, где наименьшие степени не стремятся к бесконечности. Пусть пробная функция $\varphi$ равна единице в окрестности окружности $\left(\left|z_{1}\right|=1, z_{2}=0\right)$ и равна нулю вне некоторой большей окрестности. Тогда, если вторая координата точки $а$ близка к нулю (а первая отлична от нуля), то $N\left(F_{k}, a\right) \varphi$ при достаточно больших $k$ равно $k$; если же вторая координата достаточно велика по модулю, то значение функционала равно нулю. Утверждение следствия не может выполняться.

Пример 2. Рассмотрим ту же последовательность отображений и подвергнем пространство $\left\{w_{1}, w_{2}\right\}$ какому-нибудь повороту, например, на $45^{\circ}$ (отображения задаются формулами $\left.\left\{w_{1}=\left(z_{1}^{k}+z_{2}\right) / \sqrt{2}, w_{2}=\left(-z_{1}^{k}+z_{2}\right) / \sqrt{2}\right\}\right)$. Теперь последовательность наименьших степеней стремится к бесконечности, но отображения перестали быть отображениями общего вида. Структура множества исключительных значений не изменилась.

\title{
ЛИТЕРАТУРА
}

1. Дектлрев И. М. В сб.: Многомерный комплексный анализ. Изд-во Ин-та физики СО АН СССР, Красноярск, 1985, с. 64-77. 2. Ландкоф Н. С. Основы современной теории потенциала. Наука, М., 1966. 3. Russakovskii A., Sodin M. Indiana Univ. Math. J., 44, No. 3, 851-882 (1995). 4. Russakovskii A., Shiffman B. Indiana Univ. Math. J., 46, No. 3, 897-932 (1997).

Владимирский государственный педагогический университет

Поступило в редакцию 4 декабря 1997 г. В переработанном виде 25 ноября 1998 г.

УДК $517.984,517.58$

\section{Бесконечномерные эллиптические координаты*}

\author{
(C) 1999. А. Г. Костюченко, А. А. Степанов
}

Многие конечномерные вполне интегрируемые задачи механики, например, задача о движении по геодезическим эллипсоида, задача о плоском движении материальной точки в поле притяжения двух неподвижных центров, задача Кеплера о движении точки под действием гравитационного притяжения неподвижного центра и дополнительной постоянной силы, а также ряд других задач, решаются с помощью эллиптических координат Якоби [1-3].

*Работа выполнена при поддержке РФФИ, грант № 96-01-00333 и грант поддержки научных школ № 96-15-01292. 